\title{
Synthesis of polyrotaxane-glycine conjugates with various degrees of substitution via conjugation with Boc- or Z-glycine and subsequent deprotection
}

\author{
Jun $\mathrm{Araki}^{1,3}$ and Keisuke Kagaya ${ }^{2}$
}

Two types of polyrotaxane (PR)-glycine conjugates with different degrees of substitution (DS) were prepared by $\mathrm{N}, \mathrm{N}$-carbonyldiimidazole-mediated esterification. A wide range of DS values were achieved by varying the reaction conditions, including the stoichiometric ratios of protected glycines to hydroxyls, reaction temperatures and reaction times. Varying the stoichiometric ratios gave the broadest DS control. Subsequent deprotection yielded cationic PR derivatives with different DS values. Successful deprotection of $Z$ groups was achieved only by treatment with a trifluoroacetic acid/thioanisole mixture, but was unsuccessful using conventional hydrogenation and treatment with a hydrobromic acid/acetic acid mixture. The DS values of the deprotected derivatives determined by colloidal titration agreed well with those of the protected conjugates determined by ${ }^{1} \mathrm{H}$ nuclear magnetic resonance, suggesting no removal of the pendant glycines during deprotection. The PR-glycine conjugates with higher DS values were soluble in a wide range of organic solvents, and all of the deprotected derivatives were water soluble.

Polymer Journal (2013) 45, 1081-1086; doi:10.1038/pj.2013.15; published online 27 February 2013

Keywords: amino acids; degrees of substitution (DS); deprotections; $N, N^{\prime}$-carbonyldiimidazole (CDI); polyrotaxanes

\section{INTRODUCTION}

In the last decade, a novel research area of advanced supramolecular materials science has developed as a result of investigations of various supramolecules, especially polyrotaxanes (PRs) and their derivatives, ${ }^{1-4}$ and progress in understanding their fundamental properties. ${ }^{5-7}$ A PR consisting of poly(ethylene glycol) (PEG) as an axis and $\alpha$-cyclodextrins as ring components is one of the most examined and used types of supramolecules, and has been used to create new materials such as conceptual 'slide-ring materials', ${ }^{1-3}$ promising medical devices ${ }^{4}$ and textiles with improved properties. ${ }^{8-11}$ More recently, much attention has been focused on the synthesis, characterization and use of various PR derivatives, in which different types of functional groups were incorporated onto $\mathrm{CD}$ moieties to obtain a wide range of properties. ${ }^{12-18}$ For example, the introduction of various functional groups on the PR resulted in solubility of the obtained derivatives in water and a wide variety of solvents, ${ }^{12-14}$ in contrast to the limited solubility of the unmodified PR. ${ }^{19-21}$ Water-soluble ionic PRs obtained by introduction of ionic groups can be further crosslinked to form ionic 'slide-ring gels', which have freely mobile crosslinks equipped with ionic groups, and show interesting properties, including swelling ratios up to ca 1200fold and good responsiveness to electric fields. ${ }^{14}$ Control of the degrees of substitution (DS) of these derivatives over a wide range is an important subject, as the properties of the obtained derivatives, including solvent solubility, swelling ratio of the crosslinked gels, degree of crystallinity and heat stability, are dependent on their DS values.

We have recently developed a synthesis of a novel PR-amino acid conjugate with a number of pendant amino acids on $\mathrm{CD}$ moieties by ester formation between the hydroxyls of the CDs and the carboxyls of protected amino acids, that is, tert-butyloxycarbonylglycine (Boc-Gly) and $\mathrm{N}$-benzyloxycarbonylglycine (Z-Gly), mediated by $N, N^{\prime}$-carbonyldiimidazole (CDI). ${ }^{22}$ Repeated preparations of the PR conjugates with Boc-Gly (Boc-Gly-PRs) indicated slightly different solvent solubilities of these conjugates based on subtle difference in their DS values. Further deprotection of the Boc-Gly-PRs by treatment with trifluoroacetic acid (TFA) successfully yielded cationic PR derivatives with pendant primary amino groups, without breaking the ester linkages between the PRs and Gly. However, our previous study did not achieve some important objectives, including deprotection of the conjugates with Z-Gly (Z-Gly-PRs) and control of their DS values. One of the significance results from the preparation and deprotection of different PR-amino acid conjugates with other types of protective groups is not only the

\footnotetext{
${ }^{1}$ International Young Researchers' Empowerment Center, Shinshu University, Ueda, Japan and 'Graduate School of Science and Technology, Shinshu University, Ueda, Japan ${ }^{3}$ Present address: Faculty of Textile Science and Technology, Shinshu University, Ueda, Japan.

Correspondence: Dr J Araki, Faculty of Textile Science and Technology, Shinshu University, Tokida 3-15-1, Ueda 386-8567, Japan.

E-mail: jun@shinshu-u.ac.jp

Part of this study was presented at Frontiers in Polymer Science, International Symposium Celebrating the 50th Anniversary of the Journal Polymer, at Mainz, Germany, on 7-9 June 2009.

Received 22 October 2012; revised 17 January 2013; accepted 17 January 2013; published online 27 February 2013
} 
synthesis of a cationic PR, the final product, but also the establishment of a wide variety of deprotection strategies. Establishing various deprotection routes for preparing the final cationic conjugate is of great significance, as deprotection schemes may be strictly restricted when the protected conjugates should be deprotected 'in situ' in slidering materials, that is, PR-polymer composites. ${ }^{1-3}$ For example, a previous deprotection method ${ }^{22}$ that utilizes neat TFA cannot be applied if the counterpart polymer in the slide-ring material is decomposed by TFA. Variations in protecting groups also enable selective deprotection, that is, deprotection of only specific protective groups in a single conjugate, with the other parts remaining protected. Such an 'orthogonal deprotection', namely selective deprotection, at each sequential synthetic step for different reactions has already been well explored and has provided excellent results in the field of peptide synthesis. Broad control of the DS values of the conjugates is also required to obtain cationic conjugates with various DS levels. This method may be useful for the introduction of other amino acids and/or functional peptide sequences onto PRs or other polysaccharides. $^{23,24}$

In the present study, the syntheses and characterizations of PR-glycine conjugates with a wide range of DS values were examined by varying several reaction parameters, namely, the stoichiometric ratios of the protected glycines (Boc-Gly and Z-Gly) to PR hydroxyls, reaction temperatures and reaction times. Deprotection of Z-Gly-PR, which was not attained in our previous study, ${ }^{22}$ was also examined via several different deprotection strategies. The DS values of the deprotected derivatives prepared from Boc-Gly-PR and Z-Gly-PR were determined by colloidal titration and compared with the values of the protected conjugates determined by ${ }^{1} \mathrm{H}$ nuclear magnetic resonance (NMR) spectroscopy.

\section{EXPERIMENTAL PROCEDURE}

\section{Materials}

The PR was prepared from PEG with a molecular weight of 35000 and $\alpha$-cyclodextrins, using previously reported methods. ${ }^{25,26}$ The weight-average molecular weights and polydispersity indices, determined by gel-permeation chromatography (GPC), using conditions described in measurements section, are summarized in Table 1. CDI was purchased from Tokyo Chemical Industry Co., Ltd. (Tokyo, Japan). All other chemicals were purchased from Wako Pure Chemical Industries Ltd. (Osaka, Japan). All chemicals were of reagent grade and used without any special purification.

\section{Syntheses of PR conjugates with Boc-Gly (Boc-Gly-PR) and with Z-Gly (Z-Gly-PR)}

The PR conjugates with pendant Boc-Gly or Z-Gly moieties were synthesized as described in our previous report. ${ }^{22} \mathrm{CDI}(6.55 \mathrm{mmol}, 1.06 \mathrm{~g})$ was added to a solution of $6.55 \mathrm{mmol}$ of protected glycine $(1.15 \mathrm{~g}$ of Boc-Gly or $1.37 \mathrm{~g}$ of Z-Gly) in $20 \mathrm{ml}$ of dimethylacetamide (DMAc), followed by stirring for $2 \mathrm{~h}$ at room temperature. The mixture was added to a solution of PR $(500 \mathrm{mg}$, $6.55 \mathrm{mmol}$ of $-\mathrm{OH})$ in $20 \mathrm{ml}$ of DMAc containing $6 \mathrm{wt} \%$ of lithium chloride, followed by further stirring for $24 \mathrm{~h}$ at $60^{\circ} \mathrm{C}$ under an argon atmosphere. After the reaction, the conjugates were precipitated by dropwise addition of the reaction mixture into $400 \mathrm{ml}$ of a vigorously stirred 5\% aqueous solution of sodium bicarbonate (in the case of the conjugates with Boc-Gly) or pure ethanol (in the case of the conjugates with Z-Gly), and collected by centrifugation.

As summarized in Table 1, the syntheses were performed under different conditions, that is, various stoichiometric ratios of the protected glycines to PR hydroxyls, reaction temperatures and reaction times, to yield conjugates with different DS values. The names of the obtained samples, coded based on the conditions used, are also listed in Table 1. In the repeated syntheses of Boc-Gly100-PRs under the same conditions, the DS values were typically in the range $21.3-29.4 \%$, as in our previous study. ${ }^{22}$ Repeated syntheses of Z-Gly100-PR conjugates in different batches indicated DS values in the range $18.4-23.6 \%$, corresponding to $1.65-1.94 \mathrm{mmol}$ of $\mathrm{Z}$ groups per gram of conjugates. These conjugates with slightly different DS values were treated using different deprotection protocols, as described in the following sections.

Deprotection of Boc groups from Boc-Gly-PRs

As described in our previous study, ${ }^{22}$ deprotection of the Boc-Gly-PRs was performed using a procedure involving dissolution of $300 \mathrm{mg}$ of a Boc-Gly-PR

Table 1 Changes in DS and weight-average molecular weights $\left(M_{\mathrm{w}}\right)$ of PR-glycine conjugates synthesized under different conditions

\begin{tabular}{|c|c|c|c|c|c|c|}
\hline Sample name & $\begin{array}{c}\text { Protected glycine, } \\
\text { mol eq./ }-\mathrm{OH}\end{array}$ & $\begin{array}{l}\text { Reaction } \\
\text { time, } h\end{array}$ & $\begin{array}{c}\text { Reaction } \\
\text { temperature, }{ }^{\circ} \mathrm{C}\end{array}$ & $\begin{array}{c}M_{w}, \times 10^{5} \\
(\text { polydispersity index })^{a}\end{array}$ & $D S_{N M R^{\mathrm{b}}}$ & $D S_{t i t^{c}}$ \\
\hline Starting PR & - & - & - & $1.20(1.36)$ & - & - \\
\hline Boc-Gly50-PR & 0.5 & 24 & 60 & $1.52(1.30)$ & 9.34 & 5.53 \\
\hline Boc-Gly100-PR & 1 & 24 & 60 & $1.46(1.30)$ & 23.1 & 19.3 \\
\hline Boc-Gly200-PR & 2 & 24 & 60 & $1.65(1.25)$ & 45.0 & 42.2 \\
\hline Boc-Gly300-PR & 3 & 24 & 60 & $1.70(1.31)$ & 53.2 & 50.4 \\
\hline Boc-Gly100-PR-12 h & 1 & 12 & 60 & 1.41 & 23.0 & $N D^{d}$ \\
\hline Boc-Gly100-PR-48 h & 1 & 48 & 60 & 1.53 & 35.7 & 32.3 \\
\hline Boc-Gly100-PR-40C & 1 & 24 & 40 & 1.37 & 10.3 & 9.30 \\
\hline Boc-Gly100-PR-80C & 1 & 24 & 80 & 1.53 & 36.3 & 33.8 \\
\hline Z-Gly50-PR & 0.5 & 24 & 60 & $1.27(1.36)$ & 9.30 & 8.30 \\
\hline Z-Gly100-PR & 1 & 24 & 60 & $1.44(1.36)$ & 23.6 & 22.1 \\
\hline Z-Gly200-PR & 2 & 24 & 60 & $1.73(1.34)$ & 54.9 & 53.3 \\
\hline Z-Gly300-PR & 3 & 24 & 60 & $1.91(1.33)$ & 60.6 & 58.7 \\
\hline Z-Gly100-PR-12 h & 1 & 12 & 60 & $1.29(1.53)$ & 32.3 & 31.0 \\
\hline Z-Gly100-PR-48h & 1 & 48 & 60 & $1.26(1.53)$ & 39.3 & 37.9 \\
\hline Z-Gly100-PR-40C & 1 & 24 & 40 & $1.39(1.51)$ & 11.5 & 10.6 \\
\hline Z-Gly100-PR-80C & 1 & 24 & 80 & $1.70(1.49)$ & 33.3 & 32.1 \\
\hline
\end{tabular}

Abbreviations: DS, degrees of substitution; ND, not determined; PR, polyrotaxane.

aWeight-average molecular weight and polydispersity index of the protected conjugates determined by gel-permeation chromatography (dimethyl sulfoxide/0.1 $\mathrm{M} \mathrm{LiBr}$.

aWeight-average molecular weight and polydispersity index
${ }^{b} \mathrm{DS}$ values determined by ${ }^{1} \mathrm{H}$ nuclear magnetic resonance.

CDS values determined by colloidal titration after deprotection.

${ }^{\mathrm{d}} \mathrm{ND}$ because the sample after trifluoroacetic acid treatment did not dissolve in water. 
sample in $3 \mathrm{ml}$ of TFA, stirring for $2 \mathrm{~h}$ at room temperature, precipitation of the deprotected sample by pouring the TFA solution into $50 \mathrm{ml}$ of stirred diethyl ether, collection by centrifugation and subsequent vacuum drying.

\section{Deprotection of Z-Gly-PRs by hydrogenation using an $\mathrm{H}_{2}-\mathrm{Pd} / \mathrm{C}$ system}

Z-Gly100-PR ( $373 \mathrm{mg}, 0.723 \mathrm{mmol}$ of $\mathrm{Z}$ groups, determined by ${ }^{1} \mathrm{H}$ NMR) was dissolved in $5 \mathrm{ml}$ of tetrahydrofuran (THF), followed by addition of a $10 \%$ $\mathrm{Pd} / \mathrm{C}$ catalyst $(88 \mathrm{mg})$. The sample was gently shaken for $2 \mathrm{~h}$ under a sufficient supply of $\mathrm{H}_{2}$ gas. After removal of the $\mathrm{Pd} / \mathrm{C}$ catalyst by filtration, the solution was concentrated using a rotary evaporator and further vacuum dried to yield a colorless film-like solid (170 mg).

\section{Deprotection of Z-Gly-PRs by hydrogenation using a} 1,4-cyclohexadiene-Pd/C system

Z-Gly100-PR ( $500 \mathrm{mg}, 0.970 \mathrm{mmol}$ of $\mathrm{Z}$ groups, determined by ${ }^{1} \mathrm{H}$ NMR) was dissolved in $15 \mathrm{ml}$ of dimethylformamide (DMF) followed by addition of a $10 \% \mathrm{Pd} / \mathrm{C}$ catalyst $(1.30 \mathrm{~g})$ and 1,4-cyclohexadiene $(0.744 \mathrm{~g}, 9.28 \mathrm{mmol})^{27,28}$. The mixture was stirred overnight at $25^{\circ} \mathrm{C}$ under an argon atmosphere, followed by removal of the catalyst by filtration and drying with a rotary evaporator to yield a transparent colorless film $(448 \mathrm{mg})$.

\section{Deprotection of Z-Gly-PRs by acidolysis with a mixture of hydrobromic acid and acetic acid $(\mathrm{HBr} / \mathrm{AcOH})$}

To a solution of Z-Gly100-PR ( $100 \mathrm{mg}, 0.165 \mathrm{mmol}$ of Z groups, determined by $\left.{ }^{1} \mathrm{H} \mathrm{NMR}\right)$ in $2 \mathrm{ml}$ of TFA, AcOH containing $25 \% \mathrm{HBr}(25 \% \mathrm{HBr} / \mathrm{AcOH}, 100 \mu \mathrm{l})$ was added dropwise, followed by stirring for $24 \mathrm{~h}$ at room temperature. ${ }^{29,30}$ The sample was precipitated in $50 \mathrm{ml}$ of stirred diethyl ether, collected and washed by centrifugation, and vacuum dried to yield a white solid (79.0 mg).

\section{Deprotection of Z-Gly-PRs by acidolysis with TFA/thioanisole system}

To a solution of Z-Gly100-PR ( $100 \mathrm{mg}, 0.165 \mathrm{mmol}$ of $\mathrm{Z}$ groups, determined by ${ }^{1} \mathrm{H}$ NMR $)$ in $2 \mathrm{ml}$ of TFA, thioanisole $(0.51 \mathrm{~g}, 4.13 \mathrm{mmol})$ was added dropwise, followed by stirring for $24 \mathrm{~h}$ at room temperature. ${ }^{31,32}$ The sample was precipitated into $50 \mathrm{ml}$ of stirred diethyl ether, collected and washed by centrifugation, and vacuum dried. The obtained solid was dissolved in $100 \mathrm{ml}$ of deionized water and further dialyzed against deionized water for 2 days, followed by freeze-drying to yield a white solid $(101 \mathrm{mg})$.

\section{Measurements}

The ${ }^{1} \mathrm{H}$ NMR spectra at $400 \mathrm{MHz}$ were recorded in deuterated dimethyl sulfoxide (DMSO- $d_{6}$ ) or $4 \% \mathrm{NaOD} / \mathrm{D}_{2} \mathrm{O}$ using a Bruker AVANCE spectrometer (Bruker BioSpin K.K., Yokohama, Japan) at room temperature. The chemical shifts were referenced to tetramethylsilane $(\delta=0)$ in the case of DMSO- $d_{6}$ and residual protons in the deuterated solvents $(\delta=4.70)$ in the case of $\mathrm{NaOD} / \mathrm{D}_{2} \mathrm{O}$. The latter solvent system was used to determine the DS values of the Boc-Gly-PRs and Z-Gly-PRs after complete hydrolysis of the ester linkages between the PR and the protected glycines in $\mathrm{NaOD} / \mathrm{D}_{2} \mathrm{O}$ and subsequent NMR measurements. ${ }^{22}$ The DS values of the Boc-Gly-PRs and Z-Gly-PRs were calculated from the ratio of $A_{\mathrm{Boc}}$ to $A_{\mathrm{H} 1}$ and $A_{\mathrm{Z}}$ to $A_{\mathrm{H} 1}$, respectively, where $A_{\mathrm{Boc}}, A_{\mathrm{Z}}$ and $A_{\mathrm{H} 1}$ are the integrated areas of proton signals from Boc groups (around 1.39 p.p.m.), $\mathrm{Z}$ groups (7.34 p.p.m.) and anomeric protons in the CD (around 4.8 p.p.m.), respectively. The DS values calculated from the ${ }^{1} \mathrm{H}$ NMR measurements are denoted by $\mathrm{DS}_{\mathrm{NMR}}$.

GPC of all the conjugates and colloidal titration for determination of the primary amino group contents in the deprotected samples were performed similarly to those in our previous study. ${ }^{22}$ The DS values calculated from the colloidal titrations are denoted by $\mathrm{DS}_{\text {tit. }}$. The qualitative solubilities of the obtained PR-glycine conjugates in water and in various organic solvents were visually examined by mixing $10 \mathrm{mg}$ of the conjugate with $1 \mathrm{ml}$ of solvent and subsequent shaking at room temperature.

\section{RESULTS AND DISCUSSION}

Changes in DS values of the PR-glycine conjugates synthesized under various conditions

The DS values, weight-average molecular weights and polydispersity indices of various PR-glycine conjugates synthesized under different conditions are summarized in Table 1 . The $\mathrm{DS}_{\mathrm{NMR}}$ values of the Boc-Gly-PRs varied from 9.34 to $53.2 \%$ on changing [Boc-Gly]/ [-OH] from 0.5 to 3.0, and the $\mathrm{DS}_{\mathrm{NMR}}$ values of the Z-Gly-PRs varied from 9.30 to $60.6 \%$ for the same [Z-Gly]/[-OH] range. The $\mathrm{DS}_{\mathrm{NMR}}$ values of the Boc-Gly-PRs and Z-Gly-PRs were $32.3-39.3 \%$ and 23.0-35.7\%, respectively, when synthesized using reaction times ranging from 12 to $48 \mathrm{~h}$. Variations in the reaction temperature from 40 to $80^{\circ} \mathrm{C}$ also affected the resulting $\mathrm{DS}_{\mathrm{NMR}}$ values: $11.5-33.3 \%$ for Boc-Gly-PRs and $10.3-36.3 \%$ for Z-Gly-PRs. The widest $\mathrm{DS}_{\mathrm{NMR}}$ control was observed for both Boc-Gly-PRs and Z-Gly-PRs when the [amino acid]/[-OH] ratios were varied. The use of [amino acid]/ $[-\mathrm{OH}]$ ratios higher than 3 may be effective for the synthesis of conjugates with higher $\mathrm{DS}_{\mathrm{NMR}}$ values. The increase in $\mathrm{DS}_{\mathrm{NMR}}$ values for both Boc-Gly-PRs and Z-Gly-PRs plateaued at $24 \mathrm{~h}$. With fixed reaction time of $24 \mathrm{~h}$ and [Boc-Gly]/[ $-\mathrm{OH}]$ (or [Z-Gly]/[-OH]) of $1: 1$, the $\mathrm{DS}_{\mathrm{NMR}}$ values of the conjugates increased almost linearly with increasing reaction temperature from 40 to $80^{\circ} \mathrm{C}$. These results clearly show effective control of the $\mathrm{DS}_{\mathrm{NMR}}$ values of the conjugates by varying the reaction conditions.

\section{Deprotection of Z-Gly-PRs}

Although our previous study ${ }^{22}$ achieved successful deprotection of Boc-Gly-PRs using a treatment with neat TFA to yield a deprotected cationic PR derivative, deprotection of Z-Gly-PRs was not examined. In the present study, the authors first examined the deprotection of Z-Gly-PRs by hydrogenation with a Pd/C catalyst and two sources of hydrogens, that is, $\mathrm{H}_{2}$ gas and 1,4-cyclohexadiene.

After treatment of the Z-Gly-PR sample with a combination of $\mathrm{Pd} / \mathrm{C}$ and $\mathrm{H}_{2}$ gas, the $\mathrm{Pd} / \mathrm{C}$ catalyst was removed by filtration and the THF solvent was removed by evaporation, yielding $170 \mathrm{mg}$ of solid. The ${ }^{1} \mathrm{H}$ NMR spectrum of the obtained material showed signals from aromatic protons at around 7.3 p.p.m. (data not shown), suggesting incomplete removal of the $\mathrm{Z}$ groups. GPC measurements of the obtained material also showed strong ultraviolet absorption at the elution time corresponding to the Z-Gly-PR. These results clearly indicate unsuccessful removal of $\mathrm{Z}$ groups. Only part of the sample ( $170 \mathrm{mg}$ from $373 \mathrm{mg}$ of starting sample) was recovered; the rest of the material may be adsorbed on the $\mathrm{Pd} / \mathrm{C}$ catalyst. We tried to extract the deprotected PR derivatives by washing the used $\mathrm{Pd} / \mathrm{C}$ catalyst with water and freeze-drying the washings. However, no trace of PR was observed in the ${ }^{1} \mathrm{H}$ NMR spectra of the obtained solids in DMSO- $d_{6}$. The material is presumably strongly adsorbed on the $\mathrm{Pd} / \mathrm{C}$ in the protected, water-insoluble form. One plausible reason for the unsuccessful deprotection may be the heterogeneity of the reaction system, in which a very small amount of deprotected sample, which is insoluble in THF, could fully enclose the residual Z-Gly-PRs.

Another combination of $\mathrm{Pd} / \mathrm{C}$ and 1,4-cyclohexadiene as the source of hydrogen ${ }^{27,28}$ was also examined for catalytic transfer hydrogenation of Z-Gly-PRs. Treatment of the sample with $\mathrm{Pd} / \mathrm{C}$ and 1,4-cyclohexadiene, filtration and rotary evaporation of the reaction mixture yielded a water-insoluble transparent film; the ${ }^{1} \mathrm{H}$ NMR spectrum in DMSO- $d_{6}$ showed signals from aromatic protons as well as those from the PR. GPC measurement of the obtained sample indicated that it consisted of a single ultravioletactive component with a high molecular weight comparable to that of 
the starting Z-Gly100-PR, again suggesting incomplete deprotection. The $\mathrm{DS}_{\mathrm{NMR}}$ of the obtained material $(19.6 \%)$ was almost equal to that of the starting Z-Gly100-PR (19.8\%; this is the value for a different batch from that in Table 1), suggesting almost no deprotection with this system.

An $\mathrm{HBr} / \mathrm{AcOH}$ system has also been used for the removal of $\mathrm{Z}$ groups. ${ }^{29,30}$ Deprotection of the Z-Gly-PRs by treatment with $25 \%$ $\mathrm{HBr} / \mathrm{AcOH}$ for $16 \mathrm{~h}$ at room temperature was examined in the present study. After the treatment, the transparent reaction mixture was poured into stirred diethyl ether to yield a white precipitate, which was collected by centrifugation and dried. Although the ${ }^{1} \mathrm{H}$ NMR spectrum of the obtained material in DMSO- $d_{6}$ showed the absence of signals from aromatic protons at 7.18 p.p.m. and the presence of signals from amine trifluoroacetate at 8.38 p.p.m., the ratio of the proton signals from the CDs $(3.0-4.0$ p.p.m.) to those from PEG (3.5 p.p.m.) was significantly decreased compared with that of the starting Z-Gly100-PR, suggesting a considerable loss of CD rings by significant decomposition of the PR structure. GPC measurements of the obtained material showed two elution peaks corresponding to molecular weights of ca $1.0 \times 10^{5}$ and $1.0 \times 10^{3}$, respectively. These NMR and GPC results suggest decomposition of the parent PRs as a result of treatment of the Z-Gly-PRs with $25 \% \mathrm{HBr} / \mathrm{AcOH}$ at either the terminal end-capping moiety or the $\mathrm{CD}$ rings themselves, and loss of CD moieties during the subsequent purification steps.

Another method for deprotecting $\mathrm{Z}$ groups, using a TFA/thioanisole system, ${ }^{31,32}$ involving electrophilic addition of protons to carbonyl oxygens under acidic conditions and subsequent removal of the $\mathrm{Z}$ groups by nucleophilic attack of electron-deficient carbons in benzylmethylene by sulfurs in thioanisole, was also examined for deprotection of Z-Gly-PRs. The ${ }^{1} \mathrm{H}$ NMR spectrum of the obtained material, which is shown in Figure 1a, indicates signals derived from protons in the CDs (3.0-4.0 p.p.m.) and in PEG (3.5 p.p.m.), as well as those in the primary amine trifluoroacetate (8.38 p.p.m.). Although the spectrum shows slight levels of signals around 7.0-8.0 p.p.m., which might be attributed to the protons of residual thioanisole, they disappeared in the spectrum of the same sample after purification using through dialysis against deionized water, as in Figure 1b. The GPC profile of the sample consisted of a single elution peak corresponding to a molecular weight close to that of the starting PR, suggesting no significant decomposition of the deprotected product. As in our previous study on the deprotection of Boc-Gly-PRs, ${ }^{22}$ the GPC results for the deprotected Z-Gly-PRs showed slightly lower molecular weights than that of the starting $\mathrm{PR}$, probably as a result of delayed elution resulting from attractive interactions between the GPC column and the cationic PR. The good water solubility of the deprotected sample, which must have pendant primary amino groups, is further evidence of successful deprotection, as in our previous report. ${ }^{22}$

Various Boc-Gly-PRs and Z-Gly-PRs with different DS values, listed in Table 1, were deprotected using neat TFA and TFA/thioanisole systems, respectively. The $\mathrm{DS}_{\text {tit }}$ values, as calculated from the primary amino group contents determined using colloidal titration, and summarized in Table 1, are in good agreement with the $\mathrm{DS}_{\mathrm{NMR}}$ values of the corresponding protected conjugates. These results indicate the successful deprotection of both Boc-Gly-PRs and Z-Gly-PRs with a wide range of DS values, without decomposition, to yield cationic PR derivatives with corresponding DS values. The $\mathrm{DS}_{\text {tit }}$ values of all conjugates, however, are always slightly lower than the $\mathrm{DS}_{\mathrm{NMR}}$ values, probably because the actual hydroxyl content of the starting PR might become slightly lower than our initial estimation, $13.1 \mathrm{mmol} \mathrm{g}^{-1}$, because of possible decrease in molecular weight (see Supplementary Information in detail).
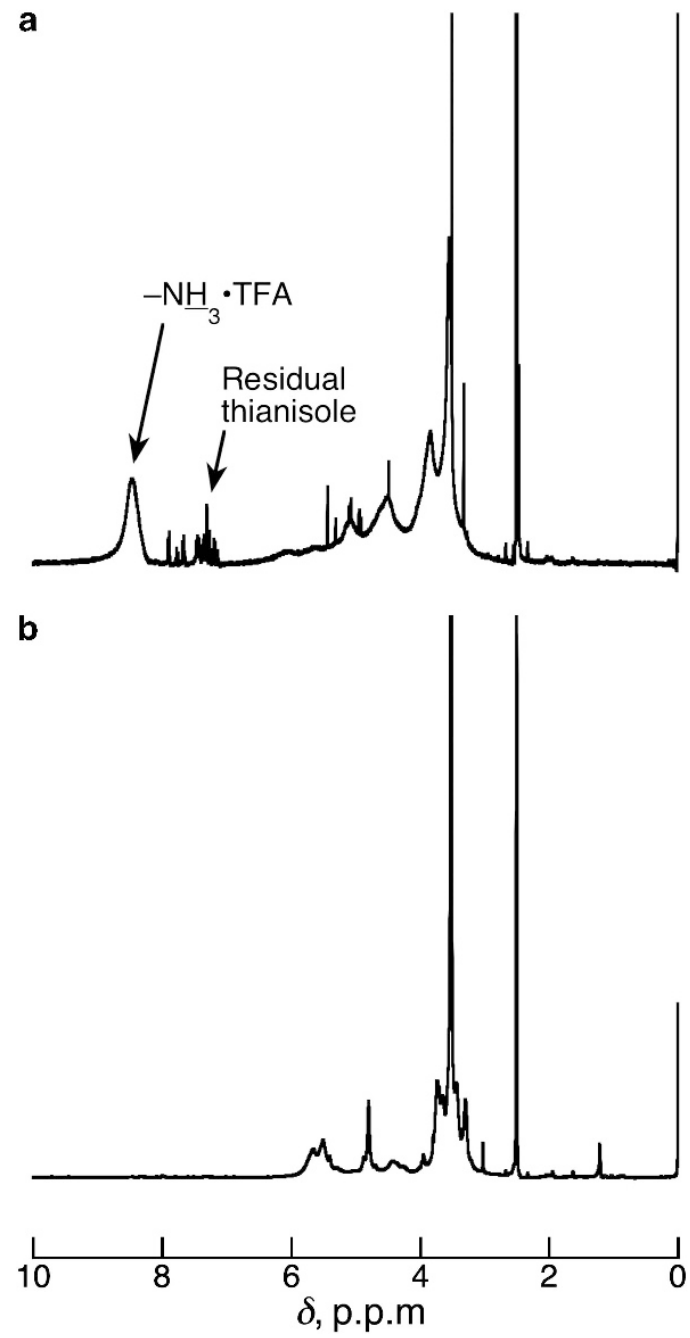

Figure $1^{1} \mathrm{H} \quad$ NMR spectra of (a) Z-Gly100-PR deprotected via TFA/thioanisole treatment and (b) the same sample dialyzed against deionized water. Both measurements were performed in DMSO- $d_{6}$.

\section{Solubility of PR-glycine conjugates with different DS values in various solvents}

The solubilities in various organic solvents and water of Boc-Gly-PRs, Z-Gly-PRs and Gly-PRs with different DS values are shown in Table 2. In contrast to the limited solubility of the starting unmodified PR, which was only soluble in DMSO, enhanced solubilities in various organic solvents, including DMAc, DMF, pyridine, methanol and THF, were observed after incorporation of pendant Boc-Gly or Z-Gly molecules. Boc-Gly-PRs with DS values above $45 \%$ and Z-Gly-PRs with DS values above $54 \%$ were also soluble in acetone and methylene chloride. All of the deprotected samples, other than deprotected Boc-Gly-PR-12 h, were soluble in water because of their cationic nature, as well as in DMSO, DMAc, DMF, pyridine and methanol. These results indicate that the solubilities of the PR-glycine conjugates can be readily controlled by varying the DS values and deprotection.

\section{CONCLUSIONS}

PR-glycine conjugates with various DS values were successfully synthesized for both Boc-Gly-PRs and Z-Gly-PRs by varying the 
Table 2 Solubilities of unmodified PR and PR-amino-acid conjugates in organic solvents and water

\begin{tabular}{|c|c|c|c|c|c|c|c|c|c|c|c|}
\hline Samples & $D S, \%^{\mathrm{a}}$ & DMSO & $D M A C$ & $D M F$ & Pyridine & Methanol & THF & Acetone & Methylene chloride & Toluene & Water \\
\hline Unmodified PR & - & $\mathrm{s}$ & i & i & i & $\mathrm{i}$ & i & i & $\mathrm{i}$ & i & i \\
\hline Z-Gly50-PR & 9.30 & $\mathrm{~s}$ & $\mathrm{~s}$ & $\mathrm{~s}$ & $\mathrm{~s}$ & $\mathrm{i}$ & i & i & i & i & i \\
\hline Z-Gly100-PR & 23.6 & $\mathrm{~s}$ & $\mathrm{~s}$ & $\mathrm{~s}$ & $\mathrm{~s}$ & $\mathrm{i}$ & $\mathrm{s}$ & i & i & i & i \\
\hline Z-Gly200-PR & 54.9 & $\mathrm{~s}$ & $\mathrm{~s}$ & $\mathrm{~s}$ & $\mathrm{~s}$ & i & $\mathrm{s}$ & $\mathrm{s}$ & $\mathrm{s}$ & i & $\mathrm{i}$ \\
\hline Z-Gly300-PR & 60.6 & $\mathrm{~s}$ & $\mathrm{~s}$ & $\mathrm{~s}$ & $\mathrm{~s}$ & $\mathrm{i}$ & $\mathrm{s}$ & $\mathrm{s}$ & $\mathrm{s}$ & $\mathrm{i}$ & $\mathrm{i}$ \\
\hline Dep-Z-Gly100-PR & $22.1^{b}$ & $\mathrm{~s}$ & $\mathrm{~s}$ & $\mathrm{~s}$ & $\mathrm{~s}$ & $\mathrm{~s}$ & $\mathrm{i}$ & $\mathrm{i}$ & $\mathrm{i}$ & $\mathrm{i}$ & $\mathrm{s}$ \\
\hline Boc-Gly50-PR & 9.34 & $\mathrm{~s}$ & $\mathrm{~s}$ & $\mathrm{~s}$ & $\mathrm{i}$ & $\mathrm{s}$ & $\mathrm{i}$ & $\mathrm{i}$ & $\mathrm{i}$ & i & i \\
\hline Boc-Gly100-PR & 23.1 & $\mathrm{~s}$ & $\mathrm{~s}$ & $\mathrm{~s}$ & $\mathrm{~s}$ & i & $\mathrm{s}$ & i & $\mathrm{i}$ & i & i \\
\hline Boc-Gly200-PR & 45.0 & $\mathrm{~s}$ & $\mathrm{~s}$ & $\mathrm{~s}$ & $\mathrm{~s}$ & $\mathrm{i}$ & $\mathrm{s}$ & $\mathrm{s}$ & $\mathrm{s}$ & i & i \\
\hline Boc-Gly300-PR & 53.2 & $\mathrm{~s}$ & $\mathrm{~s}$ & $\mathrm{~s}$ & $\mathrm{~s}$ & i & $\mathrm{s}$ & $\mathrm{s}$ & $\mathrm{s}$ & i & i \\
\hline Dep-Boc-Gly100-PR & $19.3^{b}$ & $\mathrm{~s}$ & $\mathrm{~s}$ & $\mathrm{~s}$ & $\mathrm{~s}$ & $\mathrm{~s}$ & i & i & i & i & $\mathrm{s}$ \\
\hline
\end{tabular}

Abbreviations: DMAc, dimethylacetamide; DMF, dimethylformamide; DS, degrees of substitution; i, insoluble; PR, polyrotaxane; s, soluble; THF, tetrahydrofuran.

aThe values are $D_{\text {NMR }}$ values without notice.

${ }^{\text {bDS }}{ }_{\text {tit }}$ values after deprotection.

reaction times, reaction temperatures and stoichiometric ratios of protected glycines to PR hydroxyl groups. The DS values were in the ranges $9.34-53.2 \%$ for Boc-Gly-PRs and $10.3-60.6 \%$ for Z-Gly-PRs. Changing the stoichiometric ratio was the most effective way to obtain conjugates with the widest ranges of DS values. Removal of the $\mathrm{Z}$ groups was successfully performed by treatment with a TFA/thioanisole mixture to give deprotected cationic conjugates, similar to the case of deprotection of Boc-Gly-PRs, whereas traditional methods, that is, hydrogenation using a $\mathrm{Pd} / \mathrm{C}$ catalyst and $\mathrm{HBr} / \mathrm{AcOH}$ treatment, were unsuccessful. The DS values of the protected and corresponding deprotected conjugates were in good agreement, indicating that the deprotection methods employed in the present study do not cause significant decomposition of the conjugates.

The present results are useful for further applications of cationic PR conjugates, for example, as gene carriers, ${ }^{33}$ as well as in functionalization of PR molecules with other types of amino acids and/or sequential peptides to give specific functions to PRs or slidering materials. As controlling partial deprotection of the conjugates is still difficult at present, probably owing to the relatively rapid deprotection process, especially in the case of Boc deprotection, our present results seem to be most suitable for preparing PR conjugates that have various levels of cationic group contents. In our preliminary examinations using other types of amino acids as pendant groups, all of the deprotected cationic conjugates with a DS higher than $10 \%$ were completely soluble in water, as well as in other organic solvents like DMSO, DMF, DMAc, ehanol and methanol. Further examination of PR conjugation with other amino acids, as well as of properties other than solubility of the conjugates, is now underway in our laboratory.

\section{CONFLICT OF INTEREST}

The authors declare no conflict of interest.

\section{ACKNOWLEDGEMENTS}

We greatly appreciate Professor Kousaku Ohkawa (Institute for High Polymer Research, Shinshu University) for helpful discussion on various deprotection techniques for $\mathrm{Z}$ groups. Part of this study was performed through the Program for Dissemination of Tenure-Track System, funded by the Ministry of Education, Culture, Sports, Science and Technology, Japan.
1 Ito, K. Slide-ring materials using topological supramolecular architecture. Curr. Opin. Solid State Mater. Sci. 14, 28-34 (2010).

2 Ito, K. Novel cross-linking concept of polymer network: synthesis structure, and properties of slide-ring gels with freely movable junctions. Polym. J. 39, 489-499 (2007).

3 Araki, J. \& Ito, K. Recent advances in the preparation of cyclodextrin-based polyrotaxanes and their applications to soft materials. Soft Matter 3, 1456-1473 (2007).

4 Loethen, S., Kim, J. -M. \& Thompson, D. H. Biomedical applications of cyclodextrin based polyrotaxanes. Polym. Rev. 47, 383-418 (2007).

5 Harada, A., Hashidzume, T., Yamaguchi, H. \& Takashima, Y. Polymeric rotaxanes. Chem. Rev. 109, 5974-6023 (2009).

6 Takata, T. Polyrotaxane and polyrotaxane network: supramolecular architectures based on the concept of dynamic covalent bond chemistry. Polym. J. 38, 1-20 (2006).

7 Ito, K. Novel entropic elasticity of polymeric materials: why is slide-ring gel so soft? Polym. J. 44, 38-41 (2012).

8 Araki, J., Kataoka, T., Katsuyama, N., Teramoto, A., Ito, K. \& Abe, K. A preliminary study for fiber spinning of mixed solutions of polyrotaxane and cellulose in a dimethylacetamide/lithium chloride (DMAc/LiCl) solvent system. Polymer 47, 8241-8246 (2006).

9 Katsuyama, N., Shimizu, K., Sato, S., Kuroiwa, J., Araki, J., Teramoto, A., Abe, K. \& Ito, K. Preparation of polyrotaxane fibers. Part I: influence of dope solvents on the physical properties of wet-spun polyrotaxane fibers. Textile Res. J. 80, 834-840 (2010).

10 Katsuyama, N., Shimizu, K., Sato, S., Araki, J., Teramoto, A., Abe, K. \& Ito, K. Preparation of polyrotaxane fibers. Part II: tensile properties of polyrotaxane fibers treated with two cross-linking reagents. Textile Res. J. 80, 1131-1137 (2010).

11 Sakai, Y., Ueda, K., Katsuyama, N., Shimizu, K., Sato, S., Kuroiwa, J., Araki, J., Teramoto, A., Abe, K. \& Ito, K. Fabrication and structural analysis of polyrotaxane fibers and films. J. Phys. Condens. Matter 23 article No 284108 (2011).

12 Araki, J. \& Ito, K. New Solvent for polyrotaxane. I. Dimethylacetamide/lithium chloride (DMAc/LiCl) system for modification of polyrotaxane. J. Polym. Sci. A Polym. Chem. 44, 532-538 (2006).

13 Araki, J. \& Ito, K. Polyrotaxane derivatives. I. Preparation of modified polyrotaxanes with nonionic functional groups and their solubility in organic solvents. J. Polym. Sci. A Polym. Chem. 44, 6312-6323 (2006).

14 Araki, J. Polyrotaxane derivatives. II. Preparation and characterization of ionic polyrotaxanes and ionic slide-ring gels. J. Polym. Sci. A Polym. Chem. 49, 2199-2209 (2011).

15 Kidowaki, M., Zhao, C. -M., Kataoka, T. \& Ito, K. Thermoreversible Sol-Gel transition of an aqueous solution of polyrotaxane composed of highly methylated $\alpha$-cyclodextrin and polyethylene glycol. Chem. Comm. 39, 4102-4103 (2006).

16 Kataoka, T., Kidowaki, M., Zhao, C. -M., Minamikawa, H., Shimizu, T. \& Ito, K. Local and network structure of thermoreversible polyrotaxane hydrogels based on poly(ethylene glycol) and methylated r-cyclodextrins. J. Phys. Chem. B 110, 24377-24383 (2006).

17 Araki, J., Kataoka, T. \& Ito, K. Preparation of a "sliding graft copolymer", an organic solvent-soluble polyrotaxane containing mobile side chains, and its application for a crosslinked elastomeric supramolecular film. Soft Matter 4, 245-249 (2008).

18 Araki, J., Ohkawa, K., Uchida, Y. \& Murakami, Y. Synthesis of a "molecular rope curtain": preparation and characterization of a sliding graft copolymer with grafted poly(ethylene glycol) side chains by the "grafting onto" strategy. J. Polym. Sci. A Polym. Chem. 50, 488-494 (2012). 
19 Harada, A., Li, J., Nakamitsu, T. \& Kamachi, M. Preparation and characterization of polyrotaxanes containing many threaded $\alpha$-cyclodextrins. J. Org. Chem. 58, 7524-7528 (1993).

20 Harada, A., Li, J. \& Kamachi, M. Preparation and characterization of a polyrotaxane consisting of monodisperse poly(ethylene glycol) and $\alpha$-cyclodextrins. J. Am. Chem. Soc. 116, 3192-3196 (1994).

21 Samitsu, S., Araki, J., Kataoka, T. \& Ito, K. New solvent for polyrotaxane. II. Dissolution behavior of polyrotaxane in ionic liquids and preparation of ionic liquid-containing slide-ring gels. J. Polym. Sci. B. Polym. Phys. 44, 1985-1994 (2006).

22 Araki, J., Kagaya, K. \& Ohkawa, K. Synthesis and characterization of polyrotaxaneamino acid conjugates: a new synthetic pathway for amino-functionalized polyrotaxanes. Biomacromolecules 10, 1947-1954 (2009).

23 Kuboe, Y., Tonegawa, H., Ohkawa, K. \& Yamamoto, H. Quinone cross-linked polysaccharide hybrid fiber. Biomacromolecules 5, 348-357 (2004).

24 Tonegawa, H., Kuboe, Y., Amaike, M., Nishida, A., Ohkawa, K. \& Yamamoto, H. Synthesis of enzymatically crosslinkable peptide-poly(L-lysine) conjugate and creation of bio-inspired hybrid fibers. Macromol. Biosci. 4, 503-511 (2004).

25 Araki, J., Zhao, C. -M. \& Ito, K. Efficient production of polyrotaxanes from $\alpha$-cyclodextrin and poly(ethylene glycol). Macromolecules 38, 7524-7527 (2005).

26 Araki, J. Effect of preparation conditions for poly(ethylene glycol)/cyclodextrin polyrotaxane on modes of end-capping reactions and decomposition of the yielded polyrotaxane. J. Polym. Sci. A Polym. Chem. 48, 5258-5264 (2010) (Erratum. J. Polym. Sci. A Polym. Chem. 49, 1298 (2011)).

27 Linstead, R. P., Braude, E. A., Mitchell, P. W. D., Wooldridge, K. R. H. \& Jackman, L. M. Transfer of hydrogen in organic systems. Nature 169, 100-101 (1952).

28 Felix, A. M., Heimer, E. P., Lambros, T. J., Tzougraki, C. \& Meienhofer, J. Rapid removal of protecting groups from peptides by catalytic transfer hydrogenation with 1,4-cyclohexadiene. J. Org. Chem. 43, 4194-4196 (1978).

29 Ben-Ishai, D. \& Berger, A. Cleavage of N-carbobenzoxy groups by dry hydrogen bromide and hydrogen chloride. J. Org. Chem. 17, 1564-1570 (1952).

30 Ben-Ishai, D. The use of hydrogen bromide in acetic acid for the removal of carbobenzoxy groups and benzyl esters of peptide derivatives. J. Org. Chem. 19, 62-66 (1954).

31 Kiso, Y., Ukawa, K., Nakamura, S., Ito, K., Akita, T. \& Moritoki, H. Efficient removal of protecting groups by a 'Push-Pull' mechanism. II. Deprotection of O-benzyltyrosine with a thioanisole-trifluoroacetic acid system without O-to-C rearrangements. Chem. Pharm. Bull. 28, 673-676 (1980).

32 Kiso, Y., Ukawa, K. \& Akita, T. Efficient removal of N-benzyloxycarbonyl group by a Push-Pull Mechanism using thioanisole-trifluoroacetic acid, exemplified by a synthesis of Met-enkephalin. J. Chem. Soc. Chem. Commun. 101-102 (1980).

33 Yang, C., Wang, X., Li, H., Goh, S. H. \& Li, J. Synthesis and characterization of polyrotaxanes consisting of cationic $\alpha$-cyclodextrins threaded on poly[(ethylene oxide)ran-(propylene oxide)] as gene carriers. Biomacromolecules 8, 3365-3374 (2007).

Supplementary Information accompanies the paper on Polymer Journal website (http://www.nature.com/pj) 